\title{
The concurrence of Microalbuminuria and Retinopathy with Cardiovascular Risk Factors; reliable predictors of Asymptomatic Coronary Artery Disease in Type 2 Diabetes
}

\author{
George Ioannidis ${ }^{1}$, Melpomeni Peppa ${ }^{1}$, Phivi Rontogianni ${ }^{2}$, Michael Callifronas ${ }^{1}$, \\ Christos Papadimitriou ${ }^{3}$, Georgia Chrysanthopoulou ${ }^{1}$, Lambros Anthopoulos ${ }^{3}$, \\ Matina Kesse ${ }^{2}$, Nicolaos Thalassinos ${ }^{1}$
}

${ }^{1}$ Department of Endocrinology, Diabetes and Metabolism, ${ }^{2}$ Department of Nuclear Medicine and

${ }^{3} 1^{\text {st }}$ Department of Cardiology, Evangelismos Hospital, Athens, 10676 Greece

\begin{abstract}
People with diabetes mellitus type 2 (DM2) have a greater risk for premature morbidity and mortality due to cardiovascular disease than the general population: cardiovascular disease accounts for $75 \%$ of deaths in this population group. We examined whether or not the association of clinical cardiovascular risk factors (RF) with both microalbuminuria (MA) and diabetic retinopathy (DR) constitutes reliable evidence for the existence of asymptomatic coronary artery disease (CAD), as assessed by positive myocardial thallium scintiscan using the SPECT method (Tl-scan) in patients with DM2. The study included 76 individuals with DM2 (54 men and 22 women, aged 46-70 years), with a negative history for infarction and negative clinical or ECG findings of CAD. In all patients, 3 overnight (11 pm - $7 \mathrm{am}$ ) urine collections were made for evaluation of MA. Fundoscopy after dilatation and a Tl-scan (reference method) were also carried out. In addition, blood pressure and waist/ hip ratio were measured and smoking habits were recorded. In the 35 patients with a positive Tl-scan $(46 \%)$ a higher $(\mathrm{p}<0.001)$ incidence of MA, DR, hypertension, smoking and higher waist/hip ratio were detected. Of the 16 patients with concurrent presence of MA and DR, 15 had a positive Tl-scan $(94 \%)$, whereas the Tl-scan was negative in 30/36 (83\%) patients with absence of both MA and DR. One or no cardiovascular RF in the absence of MA and DR increased the prediction of a negative Tl-scan to $100 \%$ (NPV: 1.00). Based only on history, fundoscopy and MA testing, and without resorting to expensive and laborious testing procedures, it is possible to safely distinguish patients with type 2 diabetes, who require no further investigations for asymptomatic CAD.
\end{abstract}

Key words: Asymptomatic Coronary Artery Disease, Microalbuminuria, Retinopathy, Cardiovascular Risk Factors, Diabetes Mellitus type 2 


\section{INTRODUCTION}

Diabetes mellitus is the most common metabolic disorder worldwide (approximately 5-6\% incidence in the U.S.A.) with continuously increasing incidence due to the extension of life expectancy. Type 2 diabetes mellitus (DM2) involves more than $90 \%$ of the cases of the disease, and the greatest risk for people with DM2 is the high incidence of premature morbidity and mortality due to cardiovascular disease, which accounts for $75 \%$ of deaths of which $75 \%$ are due to coronary artery disease $(\mathrm{CAD})^{1-3}$. The mortality of $\mathrm{DM} 2$ patients without known CAD is similar to the mortality of non-diabetics who have suffered a myocardial infarction ${ }^{4}$.

Based on epidemiological studies of the incidence of CAD in DM2, the American Diabetes Association (ADA), in evaluating the significance of early diagnosis of $\mathrm{CAD}$, advises testing of all DM2 patients for CAD, especially when other classical risk factors for cardiovascular disease are present. If CAD is detected, aggressive treatment of dyslipidemia and hypertension is recommended as in secondary prevention of CAD in the general population ${ }^{5}$. It is evident therefore that timely diagnosis of CAD in DM2 patients, which often remain asymptomatic for a long period, is of great importance. The most reliable non-invasive method for diagnosing asymptomatic CAD is myocardial scintiscan with thallium-201, particularly the SPECT method (single-photon emission computed tomography - Tl-scan). This method is carried out in specialized centers at considerable financial cost, factors that preclude its use as a screening test ${ }^{3,5-7}$. In recent years, several retrospective and prospective studies have shown that in diabetic and, to a lesser extent, non-diabetic Caucasian populations, the presence of microalbuminuria (MA) is associated with an increased incidence of clinical or asymptomatic $\mathrm{CAD}^{8-}$ ${ }^{12}$ and untimely deaths due to cardiovascular disease ${ }^{13,14}$. Based on these studies, it appears that MA in DM2 individuals is an indicator of vascular damage in general and represents an independent risk factor for increased morbidity and mortality due to $\mathrm{CAD}^{15-18}$. Additionally, several studies have established a correlation between Diabetic Retinopathy (DR) and increased mortality due to $\mathrm{CAD}$, while an increased incidence of DR is found in the presence of $\mathrm{MA}^{19,20}$. The purpose of the present prospective study is to evaluate whether the concurrence of MA, DR and the clas- sical risk factors (RF) for cardiovascular disease in DM2 patients may constitute a reliable indicator of the presence of asymptomatic CAD.

\section{PATIENTS AND METHODS}

This study included 76 individuals with DM2 (54 men and 22 women, aged 46-70 years), with a known duration of disease (10.5 \pm 7.5 years). Exclusion criteria were: a) history of myocardial infarction, known or electrocardiographic coronary artery disease; b) heart failure; c) diabetic nephropathy; d) uncontrolled hypertension (>180-100 $\mathrm{mmHg}$ ); e) ketoacidosis or acute dysregulation of $\mathrm{DM}$ or $\mathrm{HbA}_{1} \mathrm{c}>9.5 \%$; f) severe chronic or acute illness; and g) nephrolithiasis. In all patients, the presence of the classical cardiovascular RF (hypertension, dyslipidemia, smoking, family history of premature death due to coronary artery disease) was assessed. BMI and waist/hip ratio were recorded. Three overnight (11 pm - 7 am) urine collections over a period of three months for albumin determination were carried out. A Tl-scan (reference method) was also performed ${ }^{3,21,22}$. The presence of DR (background or proliferative) was assessed by fundoscopy following mydriasis by the same ophthalmologist. MA, measured by a radioimmunologic method, was considered present when values between 20-200 $\mu \mathrm{g} / \mathrm{min}$ in two of the three overnight urine collections ${ }^{23}$ were found. Total cholesterol, HDL cholesterol and fasting triglyceride levels were measured enzymatically. LDL cholesterol was calculated using the formula: total cholesterol - (HDL + triglycerides/5). In cases of triglyceride levels $\geq 4.5 \mathrm{mmol} / \mathrm{L}, \mathrm{LDL}$ cholesterol was not calculated. Dyslipidemia was defined as total cholesterol $\geq 5.7 \mathrm{mmol} / \mathrm{L}$ and/or HDL cholesterol $<0.90 \mathrm{mmol} / \mathrm{L}$, and/or triglycerides $\geq 2.8 \mathrm{mmol} / \mathrm{L}$ and/ or LDL cholesterol $\geq 3.36 \mathrm{mmol} / \mathrm{L}$ and/or hypolipidemic treatment. Body mass index (BMI) was calculated based on the formula Weight (Kg)/Height. Blood pressure (BP) was measured in all patients by the same person, using the same sphygmomanometer. Following a five-minute rest, BP in all patients was measured three times in a sitting position, at two-minute intervals. Hypertension was defined as an average diastolic pressure of $\geq 90 \mathrm{mmHg}$ and/or average systolic pressure $\geq 140 \mathrm{mmHg}$ and/or antihypertensive treatment. $\mathrm{HbA}_{1 \mathrm{c}}$ was determined using a high-resolution liquid chromatography method (value in non-diabetic individuals: $<6 \%)^{24}$. Creatinine was estimated using an 
automated instrument. $\mathrm{Tl}^{201}$ scintigraphy was done after IV infusion of dipyridamole (first phase), in the redistribution phase four hours later (second phase) and, when necessary, after reinjection of $1 \mathrm{mCi}$ of $\mathrm{Tl}^{201}$ (third phase).

Pharmacologic stress myocardial perfusion protocol: Each patient received $0.56 \mathrm{mg} / \mathrm{kg}$ body weight of dipyridamole by IV infusion over 4 minutes, after which a Bruce stress stage was performed. Three $\mathrm{mCi}$ of $\mathrm{Tl}^{201}$ were injected at the end of this stage while the patient continued the stress for one more minute. The collection of scintigraphic data of the first phase started immediately after this stage.

SPECT acquisition protocol: In all SPECT acquisitions a single head, large field of view, tomographic gamma camera with a general purpose collimator was used to obtain 64 projections at 30 seconds per projection over a semicircular $180^{\circ}$ arc extending from $45^{\circ}$ left posterior oblique position to the $135^{\circ}$ right anterior oblique position. Two energy windows were used, including a $30 \%$ window centered on the 68 -to $80-\mathrm{keV}$ peak and a $20 \%$ window centered on the 167 $\mathrm{keV}$ peak. Images were acquired using a $64 \mathrm{x} 64$ image matrix. Patients presenting irreversible perfusion defects in the redistribution (second phase) scintigraphy underwent a third scan one hour after reinjection of $1 \mathrm{mCi}$ of $\mathrm{Tl}^{201}$ at rest.

Evaluation of scintigraphic data: Two independent experienced nuclear physicians evaluated the scintigraphic findings using a semi-quantitative method. The myocardium was divided into 14 segments, on the horizontal and vertical axes in each patient. The parameters considered were the number of defects on the first phase image and the degree of abnormality in Tl uptake in stress and rest comparatively. Thallium uptake was thus characterized as normal (1), mildly decreased in the first phase but normal in the second phase (2), severely decreased in the first phase but normal in the second or third phase (3), and severely decreased in all three phases (4).

Evaluation of the findings of each of the three investigative procedures was carried out independently without previous knowledge of the findings of the other procedures.

\section{STATISTICAL ANALYSIS}

For the three procedures, separately and com- bined, the sensitivity, specificity, positive and negative prognostic values and statistical significance values were calculated, using the chi-square method. Average values were tested by the Student's test. Statistically significant differences were defined as $\mathrm{p}$ value $<0.05$. Data analysis was performed using the SPSS statistical program (SPSS 10.0 for Windows).

\section{RESULTS}

Pertinent characteristics of the studied subjects are presented in Table 1.

According to the findings of the Tl-scan the patients were classified into two groups (Table 1). Thirty-five patients $(46 \%)$ were classified as positive (group $\mathrm{A})$ and 41 patients as negative (group B). In group A a higher incidence of MA ( $p<0.001)$, DR $(p<0.001)$, hypertension $(\mathrm{p}<0.005)$, smoking $(\mathrm{p}<0.001)$ and higher W/HR ( $<0.001)$ was observed, whereas BMI values were comparable. Concurrent presence or concurrent absence of MA/DR was established in 52 patients. In patients with concurrent presence of MA and DR, a positive Tl-scan was obtained in 94\% (15/ 16 ), whereas in the absence of both MA and DR, a positive Tl-scan was obtained in $17 \%(6 / 36)$ of the patients (Table 2). Sensitivity (Se), specificity (Sp), positive prognostic value (PPV) and negative prognostic value (NPV) for each of the two parameters and for their concurrent presence or absence relative to the findings of the Tl-scan results are presented in Table 3.

The association of cardiovascular RF and asymptomatic CAD is presented in Table 4; the incidence of correct diagnosis based on the number of RF present is notably lower than when using the concurrent presence or absence of MA/DR. By contrast, the combined use of RF and of the concurrent presence or absence of MA/DR has a far greater diagnostic accuracy. The simultaneous absence of RF $(=1)$ and of MA/DR had a NPV:1.00 (24/24 patients), while in the simultaneous presence of RF $(>2)$ plus concurrent MA/DR the PPV was 0.78 (14/18 patients) as shown in Table 5.

\section{DISCUSSION}

The association of cardiovascular RF and asymptomatic CAD is presented in Table 4. The incidence of correct diagnosis based on the number of RF 
Table 1. Characteristics of the subjects studied, classified in Ti-201 positive and negative groups

\begin{tabular}{lccc}
\hline & Group A (Tl-scan +) & Group B (Tl-scan -) & p \\
\hline $\mathrm{n}$ & $35(46 \%)$ & $41(54 \%)$ & \\
$\mathrm{M} / \mathrm{F}$ & $27 / 8$ & $24 / 17$ & NS \\
Age (years ) & $60.3 \pm 6.9$ & $59.9 \pm 6.1$ & NS \\
Diabetes duration (years ) & $11.2 \pm 7.4$ & $9.9 \pm 7.5$ & NS \\
BMI $\left(\mathrm{kg} / \mathrm{m}^{2}\right)$ & $28.5 \pm 3.5$ & $27.7 \pm 4.0$ & $<0.001$ \\
W/H ratio & $0.95 \pm 0.08$ & $0.89 \pm 0.07$ & NS \\
HbA1c $(\%)$ & $8.1 \pm 1.2$ & $7.9 \pm 1.3$ & $<0.001$ \\
Smoking & $21 / 35(60 \%)$ & $7 / 41(17 \%)$ & $<0.001$ \\
Albumin excretion rate $(\mu \mathrm{g} / \mathrm{min})$ & $52.2 \pm 48.9$ & $18.0 \pm 30.5$ & $<0.001$ \\
Microalbuminuria & $22 / 35(63 \%)$ & $5 / 41(12 \%)$ & $<0.001$ \\
Retinopathy & $23 / 35(66 \%)$ & $7 / 41(17 \%)$ & NS \\
Dyslipidemia & $19 / 35(54 \%)$ & $19 / 41(46 \%)$ & $<0.005$ \\
Hypertension & $20 / 35(57 \%)$ & $12 / 41(29 \%)$ & \\
\hline
\end{tabular}

Table 2. Results of the Tl-scan in relation to the presence or absence of MA and DR either separately or in concurrence

\begin{tabular}{lccccc}
\hline & N & Tl-scan(+) & $\%$ & Tl-scan(-) & $\%$ \\
\hline MA +/ DR+ & 16 & 15 & 94 & 1 & 6 \\
MA -/ DR- & 36 & 6 & 17 & 30 & 83 \\
MA +/ DR - & 11 & 7 & 64 & 4 & 36 \\
MA -/ DR+ & 13 & 7 & 54 & 6 & 46 \\
\hline
\end{tabular}

Table 3. Successful prognosis of Tl-scan result by MA and DR separately and in their concurrent presence or absence

\begin{tabular}{lccc}
\hline & $\begin{array}{c}\text { Tl-scan (+) } \\
\text { VS } \\
\text { MA }\end{array}$ & $\begin{array}{c}\text { Tl-scan (+) } \\
\text { VS } \\
\text { DR }\end{array}$ & $\begin{array}{c}\text { Tl-scan (+) } \\
\text { VS } \\
\text { MA \& DR* }\end{array}$ \\
\hline $\mathrm{N}$ & 76 & 76 & 52 \\
Sensitivity & 0.63 & 0.63 & $0.71(15 / 21)$ \\
Specificity & 0.88 & 0.83 & $0.97(30 / 31)$ \\
Positive prognostic value & 0.81 & 0.76 & $0.94(15 / 16)$ \\
Negative prognostic value & 0.73 & 0.72 & $0.83(30 / 36)$ \\
\hline
\end{tabular}

*represents the concurrent presence or absence of MA and DR

present is notably lower than when using the concurrent presence or absence of MA/DR.

In contrast, the combined use of RF with the concurrent presence or absence of MA/DR has a far greater diagnostic accuracy. The simultaneous absence of RF (=1) and of MA/DR had a NPV:1.00 (24/24 patients), whereas in the simultaneous presence of $\mathrm{RF}$ (=2) and MA/DR, the PPV was 0.78 (14/18 patients),
Table 4. Tl-scan findings in the presence or absence of cardiovascular risk factors

\begin{tabular}{lccccc}
\hline & N & Tl-scan $(+)$ & \% & Tl-scan (-) & $\%$ \\
\hline$>=2$ RF & 41 & 29 & 71 & 12 & 29 \\
$<2$ RF & 35 & 6 & 17 & 29 & 83 \\
\hline
\end{tabular}

Table 5. Successful prognosis of Tl-scan result by MA/DR (concurrent presence or absence) plus cardiovascular RF

\begin{tabular}{lcc}
\hline & $\begin{array}{c}\text { Tl-scan (+) } \\
\text { vs } \\
\text { MA-/DR- } \\
\mathbf{\&} \mathbf{~ R F ~} \leq \mathbf{1}\end{array}$ & $\begin{array}{c}\text { Tl-scan }(+) \\
\text { vs } \\
\text { Ma+/dr+ } \\
\mathbf{8} \mathbf{~ R F ~} \geq \mathbf{2}\end{array}$ \\
\hline $\mathrm{N}$ & 27 & 25 \\
Sensitivity & 1.00 & 0.93 \\
Specificity & 0.92 & 0.60 \\
Positive prognostic value & 0.33 & 0.78 \\
Negative prognostic value & 1.00 & 0.86 \\
\hline
\end{tabular}

as shown in Table 5 .

The results of this study show that the concurrent presence of MA and DR in combination with cardiovascular RF can very reliably predict the existence of CAD, assessed by myocardial Tl-scan, in DM2 patients with no clinical or ECG findings of CAD.

The high incidence of asymptomatic CAD (46\%) found in our patients must be attributed to the fact that patients with many cardiovascular RF were not excluded, as well as to the relatively long known dura- 
tion of the disease (10.5 $\pm 7.5 \mathrm{yrs})$. In DM2 the incidence of asymtomatic CAD reported in the literature ranges from 9 to $57 \%^{25-27}$. This wide range in the incidence of asymptomatic CAD is attributed to differences in the groups studied with regard to age, diabetes duration, coexisting cardiovascular $\mathrm{RF}^{6,28}$ and, most importantly, to variation in the methodology used for CAD diagnosis.

In our study we used Tl-scan by the SPECT method for CAD detection, which is considered today to be the most reliable, non-invasive diagnostic test for CAD diagnosis in diabetes with specificity and sensitivity of $89 \%$ and $93 \%$, respectively, relative to coronary angiography ${ }^{21,22}$. It is to be underlined that in other studies various methods have been used for CAD diagnosis (stress test, myocardial ultrasound under stress, thallium myocardial scintigram with or without dipyridamole injection, coronary angiography), and this fact partially explains the wide variations encountered in CAD incidence in DM2.

In the present study MA was detected in $35 \%$ of the diabetics, an incidence comparable to the one reported in the literature which ranges from $25 \%$ to $40 \%{ }^{16,17,30}$. MA incidence was significantly higher in the group of patients with positive $(63 \%)$ compared to the group with negative $(12 \%)$ T1-scan ( $<0,0001)$. In an analogous study, Rutter et al found an increased incidence of CAD in diabetic patients with MA $(65 \%)$ compared to the group without MA $(40 \%)^{28}$. Furthermore, in a 7-year prospective study of diabetic patients, Panzram et al showed that an increased incidence of MA was strongly associated with increased CAD risk ${ }^{2}$. The observed strong association of MA with CAD (asymptomatic or clinical) indicates that a common pathogenetic mechanism causes vascular damage either in the small or the large vessels ${ }^{32}$, leading to micro and macroangiopathy which seem to be parallel events in DM2.

DR was detected in $39 \%$ of the patients in the present study and its incidence was higher in the diabetic patients with positive (66\%) compared to negative (17\%) T1-scan. In a similar study, Gerstein et al found that the presence of DR was associated with a 2.5-fold increased risk for asymptomatic CAD in DM2 patients $^{29}$. Furthermore, Miettinen et al found that diabetics with proliferative DR presented at a 7-year follow-up a significantly higher percentage of CAD, compared to diabetics without $\mathrm{DR}^{19}$. A large body of evidence has shown that classical risk factors for CAD are strongly associated with the presence of MA and/ or $\mathrm{DR}^{30,31}$.

In our study we showed for the first time that the concurrent presence of MA/DR had a much better positive prognostic value, for CAD detection, than that offered by the use of cardiovascular RF. In addition, the concurrent presence of MA, DR and risk factors for CAD had a much better sensitivity and specificity, as well as positive and negative prognostic value compared to the presence of each parameter separately.

In conclusion, our findings suggest that the presence or absence of risk factors for CAD, MA and DR, all of which constitute part of DM2 outpatients screening, can be used with a high level of safety as reliable markers for asymptomatic CAD diagnosis. Furthermore, by using these simple procedures clinicians can be helped to distinguish patients who need further evaluation for $\mathrm{CAD}$ diagnosis, avoiding a great psychological and economic burden. Obviously, further studies need to be made to establish the predictive value of these factors for the presence of asymptomatic CAD in DM2 outpatients.

\section{REFERENCES}

1. Rubin RJ, Altman WM, Mendelson DN, 1994 Health care expenditures for people with diabetes mellitus. J Clin Endocr Metab 78: 809.

2. Panzram G, 1987 Mortality and survival in type 2 (non insulin-dependent) diabetes mellitus. Diabetologia 30: 123-131.

3. Sniderman A, Michel C, Racine N, 1992 Heart disease in patients with diabetes mellitus. J Clin Epidemiol 45: 1357 1370.

4. Expert Panel on Detection, Evaluation, and Treatment of High Blood Cholesterol. In: Adults 2001: Executive Summary of the National Cholesterol Education Program (NCEP) Expert Panel on Detection, Evaluation, and Treatment of High Blood Cholesteroin Adults (Adult Treatment Panel III). JAMA 285: 2486-2497.

5. American Diabetes Association, 1998 Consensus Development Conference on the Diagnosis of Coronary Heart Disease in People With Diabetes. Diabetes Care 21: 15511559.

6. American Diabetes Association, 1999 Screening for Asymptomatic Coronary Artery Disease in Diabetes. Diabetes Care 22: 1393-1400.

7. Blumenthal RS, Becker DM, Moy TF, Coresh J, Wilder LB, Becker LC, 1996 Exercise thallium tomography predicts future clinically manifest coronary heart disease in a high-risk asymptomatic population. Circulation 93: 915-923. 
8. Savage S, Estacio R, Jeffers B, Schrier W, 1996 Urinary albumin excretion as a predictor of diabetic retinopathy, neuropathy, and cardiovascular disease in NIDDM. Diabetes Care 19: 1243-1248.

9. American Diabetes Association, 2003 Clinical practice recommendations - diabetic nephropathy. Diabetes Care 26: Suppl 1: 94-98.

10. Abuaisha B, Kumar S, Malik R, Boulton AJ, 1998 Relationship of elevated urinary albumin excretion to components of the metabolic syndrome in non-insulin-dependent diabetes mellitus. Diabetes Res Clin Pract 39: 9399.

11. Mattock M, Keen H, Viberti G, et al, 1998 Coronary heart disease and urinary albumin excretion rate in Type 2 (noninsulin-dependent) diabetic patients. Diabetologia 31: 8287.

12. Buggy D, Feely J, O’Sullivan C, Walsh M, 1993 Microalbuminuria and coronary heart disease in non diabetics. Postgrad Med J 69: 704-707.

13. Mattock M, Morrish N, Viberti G, Keen H, Fitzgerald A, Jackson G, 1992 Prospective study of microalbuminuria as predictor of mortality in NIDDM. Diabetes 41: 736741.

14. Schmitz A, Vaeth M, 1992 Microalbuminuria: a major risk factor in non-insulin-dependent diabetes. A 10-year follow-up study of 503 patients. Diabet Med 5: 126-134.

15. MacLeod JM, Lutale J, Marsall SM, 1995 Albumin excretion and vascular deaths in NIDDM. Diabetologia 38: 610616.

16. Dinnen SF, Hertzel C, Gerstein MD, 1997 The association of microalbuminuria and mortality in non-insulindependent diabetes mellitus. Arch Intern Med 157: 14131418.

17. Beilin J, Stanton KG, McCann VJ, Knuiman MW, Divitini ML, 1996 Microalbuminuria in type 2 diabetes: an independent predictor of cardiovascular mortality. Aust N Z J M 26: 519-525.

18. Viberti G, 1994 Prognostic significance of microalbuminuria. Am J Hypertens 7: S69-72.

19. Miettinen H, Haffner SM, Letho S, Pyorala K, Laasko M, 1996 Retinopathy predicts coronary heart disease events in NIDDM patients. Diabetes Care 19: 1445-1448.

20. Hiller R, Sperduto RD, Podgor MJ, Ferris FL, Wilson PW, 1988 Diabetic retinopathy and cardiovascular disease in type II diabetics. The Framingham Heart Study and the Framingham Eye Study. Am J Epidemiol 128: 402409.

21. Mahmarian JJ, Verani MS, 1994 Myocardial perfusion imaging during pharmacologic stress testing. Cardiol Clin 12: 223-245.

22. Maddahi J, Rodrigues E, Berman DS, Kiat H, 1994 Stateof-the-art-myocardial perfusion imaging. Cardiol Clin 12: 199-222.

23. Christansen C, Orskov C, 1994 Rapid screening PEG radioimmunoassay for quantification of pathological microalbuminuria. Diabetic Nephropathy 3: 92-97.

24. Nathan DM, 1981 Labile glycosylated hemoglobin contributes to hemoglobin A1c as measured by liquid chromatography or electrophoresis. Clin Chem 27: 1261-1263.

25. Koistinen MJ, 1990 Prevalence of asymptomatic myocardial ischemia in diabetic Subjects. Br Med J 301: 92-95.

26. Milan Study on Atherosclerosis and Diabetes (MiSAD) Group, 1997 Prevalence of unrecognized silent myocardial ischemia and its association with atherosclerotic risk factors in non insulin-dependent diabetes mellitus. Am J Cardiol 79: 134-139.

27. Zarich S, Hill T, Lewis S, Lane S, 1990 Silent myocardial ischemia and infraction in diabetics with peripheral vascular disease: assessment by dipyridamol thalium- 201 scintigraphy. Am Heart J 120: 1073-1077.

28. Rutter MK, McComb JM, Brady S, Marsall SM, 1999 Silent myocardial ischemia and microalbuminuria in asymptomatic subjects with non-insulin-dependent diabetes Mellitus. Am J Cardiol 83: 27-31.

29. Gerstein HC, Mann J, Pogue J, et al, 2000 Prevalence and determinants of microalbuminuria in high-risk diabetic and nondiabetic patients in the Heart Outcomes Prevention Evaluation Study. The HOPE Study Investigators. Diabetes Care 23: Suppl 2: 35-39.

30. Deckert T, Feldt-Rasmussen B, Borch-Johnsen K, Kofoed-Evoldsen A, 1989 Albuminuria reflects widespread vascular damage. The Steno hypothesis. Diabetologia 32: 219-226.

31. Weitzman S, Maislon M, Bodner-Fisman B, Rosen S, 1997 Association of diabetic retinopathy, ischemic heart disease, and albuminuria with diabetic treatment in type 2 diabetic patients. A population-based study. Acta Diabetol 34: 275-279. 